\title{
DESCARTES E A TANGENTE A UMA CURVA
}

\author{
DESCARTES AND THE TANGENTS TO CURVES
}

JOÃO BOSCO PITOMBEIRA DE CARVALHO ${ }^{a}$

\begin{abstract}
Resumo
A determinação das tangentes a curvas foi importante no século XVII e os matemáticos que precederam Newton e Leibniz, entre eles Roberval, Sluse, Fermat e Descartes desenvolveram várias maneiras de resolver o problema. Bem geral, o método de Descartes permite, em princípio, determinar as tangentes a qualquer curva algébrica $f(x, y)=0$, embora as dificuldades técnicas possam aumentar muito. A regra de Hudde permite reduzí-las.
\end{abstract}

Palavras-chave: Tangentes a uma curva. Descartes. Hudde.

\begin{abstract}
How to find tangents to curves was very important in the 17th Century and mathematicians who came before Newton and Leibniz, among them Roberval, Sluse, Fermat and Descartes developed techniques to tackle this problem. Descartes's method, quite general, allows one to find tangents to algebraic curves $f(x, y)=0$, even though the technical problems can increase considerably. Hudde's rule lets one significantly reduce these.
\end{abstract}

Keywords: Tangents to curves. Descartes. Hudde.

MSC2010: 001A40, 001A45

\section{Introdução}

Os gregos sabiam determinar as tangentes de algumas curvas. Nos Elementos de Euclides, é demonstrado que a tangente a um círculo é perpendicular ao raio que passa pelo ponto de tangência. Apolônio, em seu tratado sobre as cônicas, mostra como achar tangentes à elipse, hipérbole e parábola, respectivamente. Arquimedes mostrou como determinar tangentes à 
espiral. Após isso, o estudo das tangentes a curvas é retomado com vigor no século XVII, como parte da efervescência matemática que culminaria com a criação do cálculo infinitesimal por Newton e Leibniz [1]. Entre outros, Roberval, Fermat, Descartes, Huygens, Wallis, desenvolveram, independentemente, métodos para calcular tangentes. Alguns deles eram bem específicos, funcionavam para uma curva; outros permitiam resolver grande parte dos problemas relativos às tangentes das curvas algébricas, ou seja, aquelas representadas por funções algébricas $f(x, y)=0$, aquelas da forma $\sum_{i, j=0}^{k, l} a_{i j} x^{i} y^{j}$. Todos eles tiveram destino comum: seriam abandonados após a formulação abrangente do cálculo por Newton e Leibniz. Trataremos aqui do método desenvolvido por Descartes.

\section{O método das tangentes de Descartes}

Começaremos mostrando algumas retas ou segmentos de reta que surgem naturalmente no estudo da tangente a uma curva.

Figura 1: Segmentos associados à tangente de uma curva

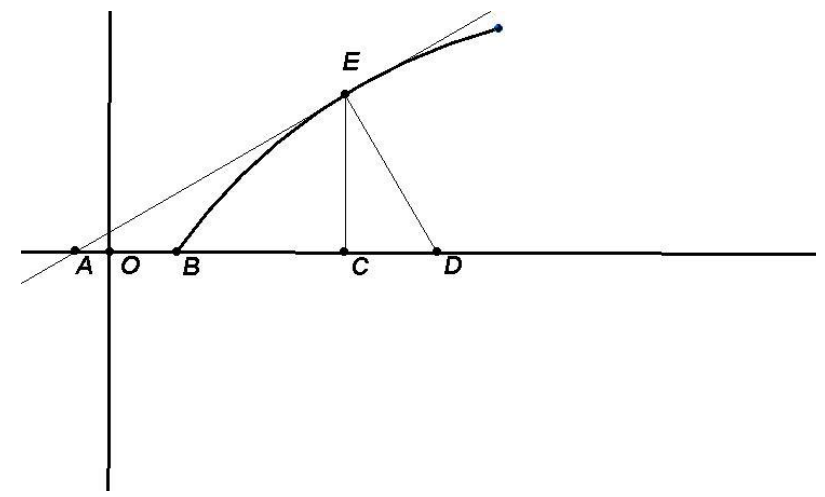

Fonte: Autoral

Na Figura 1, dada a curva que passa por $B$ e $E, A E$ é sua tangente, $A C$ é sua subtangente, $E D$ é sua normal e $C D$ é sua subnormal. É óbvio que se conhecermos um desses segmentos de reta ou retas podemos facilmente construir os outros.

O A Geometria está dividido em três partes. É na segunda delas que Descartes ataca o problema de achar a tangente a uma curva. Sobre isso, ele afirma.

Eis por que acreditaria ter posto aqui tudo o que se requer para os elementos das linhas curvas, quando, em geral, tiver dado a maneira de traçar as linhas retas que caem em ângulos retos, sobre os tais 
pontos que se quiser escolher. E ouso dizer que é este o problema o mais útil e o mais geral, não apenas o que eu saiba, mas ainda aquele que jamais desejei saber em geometria. [2, p. 53].

A afirmação de Descartes mostra bem a importância que, na época, se dava à procura de tangentes a curvas e sua crença de que seu método era superior aos dos outros matemáticos. Vejamos como ele procedeu.

Considere a curva $O E C$, da Figura 2, cuja tangente, no ponto $C$ desejamos determinar. Uma circunferência cujo centro esteja sobre o eixo $O X$, e que passe pelo ponto $C$, em geral intercepta a curva $O E C$ em dois pontos, $C$ e $E$. Há somente uma exceção, mostrada na Figura 3, quando o centro $P=(h, 0)$ da circunferência se encontra exatamente sobre a normal à curva $O E C$, pelo ponto $C$. ${ }^{1}$ Neste caso, a circunferência é tangente à curva.

Figura 2: Situação geral de uma circunferência e suas interseções com uma curva

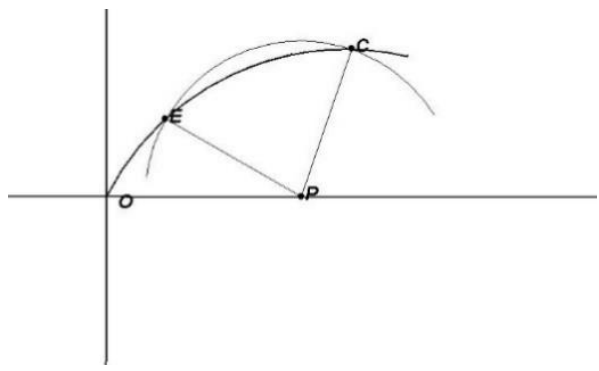

Fonte: Autoral

Se conseguirmos determinar as coordenadas do ponto $P$, ou seja, determinar $h$, é fácil achar a tangente à curva, por $C$, pois ela será perpendicular à normal pelo mesmo ponto.

Figura 3: Circunferência tangente a uma curva

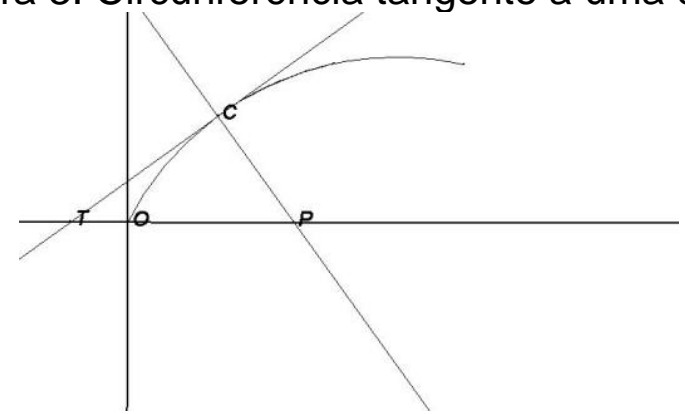

Fonte: Autoral

\footnotetext{
${ }^{1}$ Descartes ignora completamente o caso em que a curva apresenta cúspides, o que acontece, por exemplo, com algumas curvas algébricas de terceira ordem, como mostrado por Newton, que as classificou inicialmente, depois complementado por Euler. As curvas com que Descartes trabalha no A Geometria, as cônicas e as curvas que surgem na solução do problema de Pappus não têm tal comportamento.
} 


\section{Exemplo de aplicação do método de Descartes}

Vejamos como determinar o ponto $P$ no caso em que a equação da curva é $y=\sqrt{x}$. Sejam $\left(a^{2}, a\right)$ as coordenadas do ponto $C, r$ o raio da circunferência, e $(h, 0)$ as coordenadas do ponto $P$. Então, a equação da circunferência é

$$
(x-h)^{2}+y^{2}=r^{2}
$$

ou seja,

$$
x^{2}+y^{2}-2 h x+h^{2}-r^{2}=0 .
$$

Os pontos de intersecção da curva com a circunferência são achados resolvendo o sistema

$$
\left\{\begin{array}{c}
x^{2}+y^{2}-2 h x+h^{2}-r^{2}=0 \\
y=\sqrt{x}
\end{array}\right.
$$

Eliminando $y$, obtemos a equação do segundo grau

$$
x^{2}+(1-2 h) x+\left(h^{2}-r^{2}\right)=0
$$

Em geral, esta equação tem duas soluções para $x$. Sabemos que a circunferência e a curva se cortam no ponto $\left(a^{2}, a\right)$, ou seja, $x=a^{2}$ é uma das raízes da equação. Desejamos que ela seja a única raiz, ou seja, que $x=a^{2}$ seja uma raiz dupla. Para que isto aconteça, devemos ter

$$
x^{2}+(1-2 h) x+\left(h^{2}-r^{2}\right)=\left(x-a^{2}\right)^{2} .
$$

Desenvolvendo o lado direito e comparando os coeficientes, teremos

$$
1-2 h=-2 a^{2} \rightarrow h=a^{2}+\frac{1}{2}
$$

Assim, a circunferência de centro $\left(a^{2}+\frac{1}{2}, 0\right)$ será tangente ao gráfico de $y=\sqrt{x}$ no ponto $\left(a^{2}, a\right)$.

O método de Descartes, exposto no La Géométrie é extremamente elegante. Em princípio, ele funciona para curvas descritas por equações algébricas $f(x, y)=0$. No entanto, rapidamente ele se torna tecnicamente complicado. Isso será visto na próxima seção.

\section{A tangente à curva $y=x^{3}$}


Por exemplo, se tentarmos aplicá-lo para encontrar a tangente à curva de equação $y=x^{3}$, e que passa pelo ponto de coordenadas $\left(a, a^{3}\right)$, vemos que a situação se torna tecnicamente bem mais difícil.

Com efeito, devemos resolver o sistema

$$
\left\{\begin{array}{c}
y^{2}+x^{2}-2 h x+h^{2}-r^{2}=0 \\
y=x^{3}
\end{array}\right.
$$

Assim, temos que

$$
x^{6}+x^{2}-2 h x+h^{2}-r^{2}=0
$$

Se quisermos achar a tangente que passa pelo ponto $\left(a, a^{3}\right)$, esta equação deverá ter uma raiz dupla para $x=a$.

Assim, podemos escrever

$$
x^{6}+x^{2}-2 h x+h^{2}-r^{2}=(x-a)^{2}\left(x^{4}+B x^{3}+C x^{2}+D x+F\right) .
$$

Desenvolvendo o lado direito obtemos

$$
\begin{aligned}
& x^{6}+x^{2}-2 h x+h^{2}-r^{2}=x^{6}+(B-2 a) x^{5}+\left(a^{2}-2 a B+C\right) x^{4} \\
& +\left(a^{2} B-2 a C+D\right) x^{3}+\left(a^{2} C-2 a D+F\right) x^{2}+\left(a^{2} D-2 a F\right) x+a^{2} F .
\end{aligned}
$$

Comparando os coeficientes, obtemos o sistema

$$
\begin{aligned}
(B-2 a) & =0 \\
\left(a^{2}-2 a B+C\right) & =0 \\
\left(B a^{2}-2 a C+D\right) & =0 \\
\left(C a^{2}-2 a D+F\right) & =1 \\
\left(D a^{2}-2 a F\right) & =-2 h \\
a^{2} F & =h^{2}-r^{2}
\end{aligned}
$$

Com a equação (1) obtemos

$$
B=2 a \text {. (7) }
$$

Usando (2) e (7) temos

$$
a^{2}-2 a(2 a)+C=0 \rightarrow C=3 a^{2} .
$$

Utilizando estes valores de $B$ e $C$ em (3) obtemos

$$
a^{2}(2 a)-2 a\left(3 a^{2}\right)+D=0
$$

o que acarreta

$$
D=4 a^{3} .
$$

Usando todos estes valores em (4) obtemos

$$
a^{2}\left(3 a^{2}\right)-2 a\left(4 a^{3}\right)+F=1 \rightarrow F=1+5 a^{2}
$$

Assim, por meio de (5) chegamos a 


$$
a^{2}\left(4 a^{3}\right)-2 a\left(1+5 a^{4}\right)=-2 h \rightarrow h=a+3 a^{5},
$$

Ou seja, as coordenadas do centro do círculo tangente são $\left(a+3 a^{5}, 0\right)$.

Estes valores nos permitem escrever que

$$
x^{6}+x^{2}-2 h x+h^{2}-r^{2}=(x-a)^{2}\left(x^{4}+2 a x^{3}+3 a^{2} x^{2}+4 a^{3} x+1+5 a^{4}\right) \text {. }
$$

Você observa que os cálculos realmente podem se tornar complicados. Como diminuir o trabalho envolvido na aplicação do método de Descartes para achar tangentes a curvas? É o que veremos na próxima seção.

\section{Schooten e Hudde}

As dificuldades técnicas que surgem na utilização do método de Descartes diminuem bastante se usarmos a chamada "regra de Hudde", devida ao matemático holandês Hudde, que participou ativamente do círculo de matemáticos reunidos em torno de van Schooten.

Frans van Schooten (1615-1660) foi um matemático competente, lembrado principalmente por ter contribuído fortemente para a difusão das ideias matemáticas de Descartes. Ele foi professor de matemática na Universidade de Leiden e dentre seus alunos contam-se Christiaan Huygens, Johann van Waveren Hudde e René de Sluze [4]. Já em 1649 publicou uma tradução do La Géométrie para o latim, a língua culta da Europa, na época. Com ajuda de seus colegas matemáticos aumentou aos poucos essa tradução, adicionando-lhe apêndices visando a completá-la e a torná-la mais compreensível. Assim, como o latim era a língua dos cientistas da época, as ideias de Descartes puderam se difundir mais facilmente por toda a Europa. A edição do La Géométrie em dois volumes, de 1659 e 1661 respectivamente, contém apêndices de Jan de Witt, Johann Hudde e Hendrick van Heuraet. Foi esta a edição que Isaac Newton comprou e leu.

Jan (ou Johann) Hudde (1628 - 1704) formou-se em Direito, e seu envolvimento com a matemática provavelmente se encerra em 1663, data após a qual não se conhece em suas atividades nada relacionado à matemática, tendo ele se dedicado a partir daí à política, chegando a ser prefeito de Amsterdam. Em 1657 ele se correspondeu com Sluse, Huygens e Schooten sobre quadraturas, tangentes e centros de gravidade de curvas algébricas [3]. Haas considera Hudde o mais brilhante dos alunos de Schooten, tendo sido elogiado 
por Leibniz, que, em 1697, escreveu que somente se poderia esperar uma solução do problema da Braquistóocrona por Newton, L'Hospital, os Bernoullis e Hudde caso este último não tivesse abandonado tais pesquisas há muito tempo [3].

\title{
6. A regra de Hudde
}

No apêndice, em forma de carta, à edição citada de van Schooten, Hudde afirmou que

\begin{abstract}
Se em uma equação duas de suas raízes são iguais, e ela for multiplicada por uma progressão aritmética arbitrária, obviamente 0 primeiro termo da equação pelo primeiro termo da progressão, 0 segundo termo da equação pelo segundo termo da progressão e assim sucessivamente, afirmo que o produto será uma equação em que uma das acima mencionadas raízes se encontrará.
\end{abstract}

Em outras palavras, suponha que os números $p+q i$ estão em progressão aritmética, para $i=0,1, \cdots, n-2$. Então, o polinômio

$$
F(x)=\sum_{i} a_{i} x^{i}
$$

tem uma raiz dupla no ponto $e$ se e somente se o polinômio

$$
F^{*}(x)=\sum_{i} a_{i}(p+q i) x^{i}
$$

tem uma raiz simples em $e$.

Hudde dá uma demonstração para um polinômio de grau 5 , mas que funciona para qualquer grau, como veremos.

\section{Demonstração da regra de Hudde}

Demonstremos agora a regra de Hudde.

Suponha que $e$ é uma raiz dupla do polinômio $f(x)=0$. Então,

$$
\begin{gathered}
f(x)=(x-e)^{2}\left(c_{0}+c_{1} x+c_{2} x^{2}+\cdots+c_{n-2} x^{n-2}\right) \\
=(x-e)^{2}\left(\sum_{k=0}^{n-2} c_{k} x^{k}\right) \\
=\sum_{k=0}^{n-2} c_{k}\left(x^{k+2}-2 e x^{k+1}+e^{2} x^{k}\right)
\end{gathered}
$$


Se esta equação for multiplicada termo a termo por uma progressão aritmética $p+q k$, na qual $p$ e $q$ são números inteiros, e $k=0,1,2, \cdots, n-2$, obtemos o polinômio

$$
\begin{aligned}
& g(x)= \sum_{k=0}^{(n-2)} c_{k} x^{k}\left\{(p+q(k+2)) x^{k+2}-2 e(p+q(k+1)) x^{k+1}+e^{2}(p\right. \\
&\left.+q k) x^{k}\right\} \\
&=\sum_{k=0}^{(n-2)} c_{k}(p+q k)\left(x^{2}-2 e x+b^{2}\right)+\sum_{k=0}^{(n-2)} c_{k} 2 q\left(x^{(k+2)}-e b x^{(k+1)}\right) \\
&=(x-e)^{2} \sum_{k=0}^{(n-2)} c_{k}(p+q k) x^{k}+2 q(x-e) \sum_{k=0}^{(n-2)} c_{k} x^{k+1}
\end{aligned}
$$

e vemos obviamente que $e$ é uma raiz de $g(x)$.

Uma demonstração moderna da regra de Hudde seria a seguinte.

É fácil ver que

$$
g(x)=p f(x)+q x f^{\prime}(x) .
$$

Se $f(x)=(x-a)^{2} h(x)$, temos

$$
f^{\prime}(x)=2(x-a) h(x)+(x-a)^{2} h^{\prime}(x) .
$$

Assim, se $a$ é uma raiz dupla de $f(x)$, então $a$ é também uma raiz de $f^{\prime}(x)$.

Um exemplo tornará claro o funcionamento da regra.

\section{Exemplo de funcionamento da regra de Hudde}

Considere o polinômio²

$$
x^{3}-4 x^{2}+5 x-2=0
$$

e a progressão aritmética $3,2,1,0$.

\begin{tabular}{cccc}
\multicolumn{4}{r}{ Escrevamos o procedimento como segue } \\
$x^{3}$ & $-4 x^{2}$ & $+5 x$ & -2 \\
3 & 2 & 1 & 0 \\
\hline $3 x^{3}$ & $-8 x^{2}$ & $5 x$ &
\end{tabular}

Então, a regra de Hudde afirma que $e$ é raiz dupla de

$$
x^{3}-4 x^{2}+5 x-2=0
$$

somente se $3 x^{3}-8 x^{2}+5 x$ tem a raiz $e$.

\footnotetext{
${ }^{2}$ Vê-se facilmente que este polinômio pode ser fatorado como $(x-2)(x-1)^{2}$.
} 
Ora, como é fácil verificar

$$
3 x^{3}-8 x^{2}+5 x=x(x-1)(3 x-5)
$$

E vemos assim que $x=1$ é raiz simples de $3 x^{3}-8 x^{2}+5 x=0$ e dupla de $x^{3}-4 x^{2}+5 x-2=0$.

Mas, como isso ajuda a resolver o problema de determinar a tangente a uma curva? É o que veremos na próxima seção.

\section{A regra de Hudde e a determinação de tangentes}

O que esta regra tem a ver com o problema de facilitar os cálculos na utilização do método de Descartes para a determinação das tangentes às curvas? Isso acontece porque a escolha judiciosa da progressão aritmética permite reduzir consideravelmente os cálculos envolvidos.

Relembremos o que foi feito na seção 4 para achar a tangente a $y=x^{3}$, quando chegamos à equação

$$
x^{6}+x^{2}-2 h x+h^{2}-r^{2}=0,
$$

com a qual temos que determinar $h$ e $r$.

Usemos o método de Hudde para determinar suas raízes múltiplas. Para isso, escolhamos a progressão aritmética $6,5,4,3,2,1,0$ e disponhamos 0 procedimento no seguinte quadro

$$
\begin{array}{lcccccc}
x^{6}+0 x^{5} & +0 x^{4} & +0 x^{3} & +x^{2} & -2 h x & +h^{2}-r^{2} \\
6 & 5 & 4 & 3 & 2 & 1 & 0 \\
\hline 6 x^{6}+0 x^{5} & +0 x^{4} & +0 x^{3} & 2 x^{2} & -2 h x & +0
\end{array}
$$

Desta maneira, obtivemos, com a aplicação da regra de Hudde, o polinômio

$$
6 x^{6}+2 x^{2}-2 h x=0,
$$

que nos permite facilmente calcular o valor de $h$ :

$$
h=\frac{6 x^{5}+2 x}{2} \text {. }
$$

Como estamos procurando determinar a tangente no ponto $\left(a, a^{3}\right)$, chegamos a 


$$
h=\frac{6 a^{5}+2 a}{2}=3 a^{5}+a,
$$

resultado que coincide com o que obtivemos anteriormente e vemos que nosso trabalho foi muito facilitado.

\section{Agradecimentos}

Agradecemos à FAPERJ pelo apoio que permitiu a elaboração deste trabalho e ao revisor, que muito contribuiu para melhorá-lo.

\section{Referências}

[1] BARBIN, Evelyne. La Révolution Mathématique au XVII Siècle. Paris: Ellipses, 2006.

[2] DESCARTES, René. A Geometria (tradução e comentários por Bruna $F$. Barbosa, Clediane M. da Silva, Filipe B. Brant e Raquel A. Sapunaru). 2a edição. São Paulo: Livraria da Física, 2017.

[3] HAAS, Karl Heinz. Jan Hudde. In: GILLISPIE, Charles Coulston (ed.) Dictionary of Scientific Biography, v. 6, pp. 536-538. New York: Charles Scribner's Sons, 1981.

[4] HOFMANN, J. E. Frans van Schooten. In: GILLISPIE, Charles Coulston (ed.) Dictionary of Scientific Biography, v. 12, pp. 205-207. New York: Charles Scribner's Sons, 1981. 